\title{
The atypical homeoprotein Pbx1a participates in the axonal pathfinding of mesencephalic dopaminergic neurons
}

\author{
Paola Sgadò ${ }^{1,2^{*}}$, Elisabetta Ferretti ${ }^{3 \dagger}$, Daniel Grbec ${ }^{2 \dagger}$, Yuri Bozzi ${ }^{1,4}$ and Horst H Simon ${ }^{2}$
}

\begin{abstract}
Background: The pre B-cell leukemia transcription factor 1 (Pbx1) genes belong to the three amino acid loop extension family of homeodomain proteins that form hetero-oligomeric complexes with other homeodomain transcription factors, thereby modulating target specificity, DNA binding affinity and transcriptional activity of their molecular associates.

Results: Here, we provide evidence that $P b \times 1$ is expressed in mesencephalic dopaminergic neurons from embryonic day 11 into adulthood and determines some of the cellular properties of this neuronal population. In Pbx1-deficient mice, the mesencephalic dopaminergic axons stall during mid-gestation at the border between diand telencephalon before entering the ganglionic eminence, leading to a loose organization of the axonal bundle and partial misrouting. In Pbx1-deficient dopaminergic neurons, the high affinity netrin-1 receptor, deleted in colon cancer (DCC), is down-regulated. Interestingly, we found several conserved Pbx1 binding sites in the first intron of $D C C$, suggesting a direct regulation of DCC transcription by Pbx1.
\end{abstract}

Conclusions: The expression of $P b \times 1$ in dopaminergic neurons and its regulation of DCC expression make it an important player in defining the axonal guidance of the midbrain dopaminergic neurons, with possible implications for the normal physiology of the nigro-striatal system as well as processes related to the degeneration of neurons during the course of Parkinson's disease.

Keywords: Axonal outgrowth, neurodegenerative disease, Prep1, substantia nigra, transcription factors, ventral tegmentum

\section{Background}

Pre B-cell leukemia transcription factor 1 (Pbx1) encodes a transcription factor, belonging to the $\mathrm{PBC}$ ( $\mathrm{Pbx} 1$ to 4$)$ subclass of the three amino acid loop extension (TALE) proteins characterized by an atypical homeodomain [1]. Studies of the Pbx proteins and their Drosophila homo$\log$ Extradenticle (exd) revealed that they form stable complexes with other homeodomain transcription factors, such as Hox and Engrailed, as well as other nonhomeodomain proteins [2]. The interaction with $\mathrm{Pbx}$

\footnotetext{
*Correspondence: sgado@science.unitn.itl

${ }^{\dagger}$ Equal contributors

'Laboratory of Molecular Neuropathology, Centre for Integrative Biology (CIBIO), University of Trento, Trento, Italy

${ }^{2}$ Interdisciplinary Center of Neuroscience, Department of Neuroanatomy, University of Heidelberg, Heidelberg, Germany

Full list of author information is available at the end of the article
}

modulates the target selectivity, the DNA binding affinity and the transcriptional activity of the associated homeoproteins [3]. An example of the modulation of transcriptional activity by the $\mathrm{Pbx}$ transcription factors is the regulation of Fibroblast growth factor 8 (Fgf8) expression by the Engrailed transcription factors. A highly conserved region in the large intron of the Fgf8 gene contains an Engrailed/Pbx binding site. This part of the enhancer increases transcriptional activity by three to fourfold in the presence of embryonic nuclear extract containing the Engrailed proteins and Pbx1, and point mutations in the binding site inactivate it [4].

$P b x$ loss of function phenotype is to a large part a reflection of the phenotypic alterations observed after functional ablation of the associated molecular partner. In Drosophila, for example, embryos lacking exd (zygotic or maternal) show the typical homeotic transformations

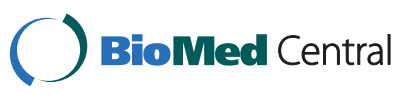


in the thoracic and abdominal segments that resemble the loss of function phenotypes of the Hox genes cooperating with exd, although their expression is unaltered [5]. In mammals, the correlation between phenotypes of mutants deficient for $P b x 1$ and null mutants for the molecular partners is not as evident [6]. $P b x$ genes have been implicated in development of the skeleton [7], pancreas [8], kidney, adrenal glands [9,10], thymus [11], spleen [12] and in hematopoiesis [13]. A number of homeodomain transcription factors play a major role in the development of all of these tissues and organs systems and may act as cofactors for $P b x$ genes.

We have previously demonstrated that the Engrailed genes are required for survival of the mesencephalic dopaminergic (mesDA) neurons [14,15]. The survival function of Engrailed genes is unique to these neurons and is not shared with other neuronal populations expressing the genes, like the cerebellar granule cells $[16,17]$ or the V1 interneurons in the spinal cord [18]. The cooperative binding to $\mathrm{Pbx} 1$ protein has already been shown to modulate the regulation of Fgf8 expression by the Engrailed genes, a crucial factor for the development of the mesDA neurons $[4,19,20]$. We therefore hypothesized that cooperative binding to $\mathrm{Pbx}$ proteins may modulate the target selectivity of the Engrailed genes in mesDA neurons.

We examined the expression and function of the $P b x$ genes in this neuronal population during development and show here that a splicing variant of $P b \times 1, P b \times 1 a$, and one of the Prep genes, Prep1, are expressed by mesDA neurons. Furthermore, our analysis of $P b x 1 \mathrm{mu}-$ tant mice demonstrates a role of $P b x 1$ in axon guidance through the regulation of the netrin-1 receptor, deleted in colon cancer $(D C C)$. Interestingly, despite increasing evidence of a cooperative function of Engrailed and $\mathrm{Pbx}$ transcription factors in vertebrates development $[4,21]$, we could not find a 1:1 correlation between Engrailed and Pbx1 mutants' phenotype. In our case, a more detailed analysis of different single and compound mutants might be necessary to confirm our original hypothesis of a Pbx/Engrailed functional cooperative binding playing a significant role in the development and survival of mesDA.

\section{Results}

Pbx 1 expression in mesencephalic dopaminergic neurons

To examine expression of the $P b x$ family members in mesDA neurons, we performed in situ hybridization on midbrain sections of various ages. We restricted our analysis to those family members, $P b x 1,2$ and 3, which are expressed in the brain [22]. Of note, only $P b x 1 a$, a splicing variant of $P b x 1$, colocalized with tyrosine hydroxylase $(T H)$, the key enzyme of dopamine synthesis (Figure 1). At embryonic day (E) 11, Pbx1a was expressed in the entire developing midbrain neuroepithelium. Ventrally on the pial site, an elevated signal was observable that overlapped with $\mathrm{TH}$ in the parallel section, suggesting co-expression (Figure 1A, B). At E14, Pbx1a expression was more restricted and now clearly overlapped with TH (Figure 1C, D). The staining with a pan-Pbx antibody at the same embryonic stage revealed that each $\mathrm{TH}$-positive cell body in the midbrain possessed a Pbx1-positive nucleus (Figure 1I-K). At postnatal ages, $P b x 1 a$ expression was decreased in intensity and disappeared in many brain regions, but remained at high levels in all mesDA neurons (for the adult see Figure $1 \mathrm{~N}, \mathrm{O})$. Furthermore, a double immunostaining using the pan-Pbx antibody and an antibody against $\beta$ galactosidase to detect the Engrailed1 (En1) reporter LacZ [23] revealed that Pbx1a is co-expressed with En1 in these neurons (for the adult see Figure 2A-E). We also detected by in situ hybridization a diffuse Pbx3 RNA signal in the ventral midbrain from E14 into the adult (Figure 1G), but a Pbx3 specific antibody on wild type was unable to detect any $\mathrm{Pbx} 3$ protein in mesDA neurons (Figure 1L, M). However, Pbx3 protein was detectable in other brain regions, like for example the raphe nucleus (Figure 1L, M insert).

\section{Pbx1 sub-cellular localization}

The activity of PBC proteins is in part regulated by nuclear import, which is mediated by dimerization with homeoproteins of the MEINOX (MEIS and KNOX) subclass, or by phosphorylation [24-27]. The MEIS subfamily of TALE proteins includes the products of the vertebrate Meis1-3, while the PREP subfamily includes the vertebrate Prep1 and Prep2. Exd and Pbx proteins have been shown to require MEIS/PREP for their nuclear import in specific cell contexts, such as limb mesenchymal cells in vertebrates or limb imaginal disc cells in flies [28-32].

Since the Pbx1a protein was localized in the nucleus of mesDA neurons, we investigated whether any of the Meis genes are also expressed in these neurons. We found Meis1, Meis2 and Meis3 expression in telencephalon, diencephalon, midbrain and hindbrain as previously described [33], but none of them in mesDA neurons (Figure 3A-D). Despite previous reports of a ubiquitous expression of Prep1 in the developing brain from as early as E7.5, we detected by immunohistochemistry a specific Prep1 domain in the ventral midbrain, co-localized with TH (Figure 3E-I), indicating that the nuclear transport of the Pbx1 protein is likely achieved in this neuronal population by molecular association with Prep1. 

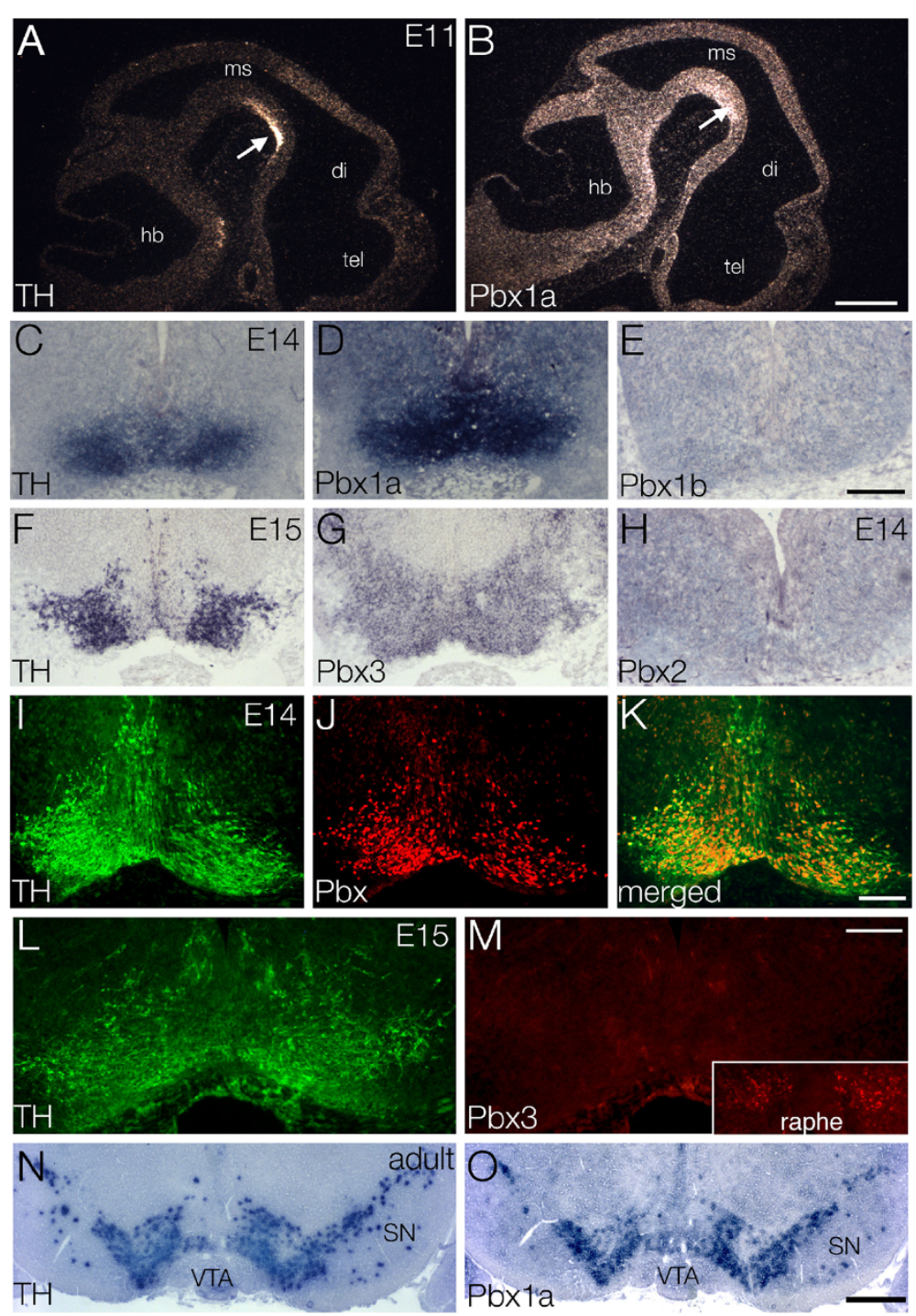

Figure 1 Expression of Pbx1a in mesencephalic dopaminergic neurons. In situ hybridization using ${ }^{35} \mathrm{~S}-(\mathrm{A}, \mathrm{B})$ and digoxigenin-riboprobes (C$H, N, O)$ against TH (A, C, F, N), Pbx1a (B, D, O), Pbx1b (E), Pbx2 (H) and Pbx3 (G) on E11 sagittal (A, B) and coronal sections of E14 (C, D, E, H), E15 $(\mathrm{F}, \mathrm{G})$ and adult $(\mathrm{N}, \mathrm{O})$ mice. Fluorescent double labeling on coronal sections of E14 (I-K) and E15 (L, M) embryos using antibodies against TH (I, L) pan-Pbx (J) and Pbx3 (M). (A, B) At E11, TH expression is confined to mesDA neurons on the pial surface of the ventral mesencephalon (arrow) (A). The parallel section reveals the full extent of the Pbxla expression with an elevated signal at the position of mesDA neurons (arrow) (B). (C-H) Pbx members' expression in the ventral midbrain. Pbx1a (D) co-localizes with TH (C). Pbx 16 (E) and Pbx2 (H) are not detectable in the ventral midbrain whereas Pbx3 (G) shows a diffused signal partially overlapping with TH (F). (I-K) Immunohistochemical double labeling using TH (I, green) and pan-Pbx (J, red) antibodies reveals the co-expression of Pbx1 and TH in mesDA neurons (K). (L, M) Double immunohistochemistry using $\mathrm{TH}(\mathrm{L}$, green), and Pbx3 (M, red) antibodies shows no detectable Pbx3 protein in the ventral midbrain. Insert: Pbx3 positive staining in raphe nucleus. (N, O) Expression of Pbxia in the adult co-localizes with TH in ventral tegmental (VTA) and nigral (SN) dopaminergic neurons.

Telencephalon (tel), diencephalon (di), mesencephalon (ms), hindbrain (hb). Dorsal to the top, A and B rostral to the right. Scale bars A-B, N, $\mathrm{O}=500 \mu \mathrm{m}, \mathrm{C}-\mathrm{H},=200 \mu \mathrm{m}$, and $\mathrm{I}-\mathrm{M}=100 \mu \mathrm{m}$. mesDA: mesencephalic dopaminergic.

\section{Analysis of $P b x 1$ mutant mice}

In order to investigate the role of Pbxla in mesDA neurons, we analyzed homologous recombinant mutant mice null for Pbx1 [7]. Up to E15.5, when the mutant mice die, mid/hindbrain morphology and the distribution of mesDA neurons appeared normal (Figure 4A, B); however, the mutants showed aberrant mesDA axonal projections. In the E13 whole mount preparations, the wild type mesDA neurons extended their axons deep into the ganglionic eminence [34] (Figure 4C, C'), whereas in $P b x 1$ null mutants, the DA axons stopped growing at the border between tel- and diencephalon, and defasciculated (Figure 4D, D'). One day later, at E14, some of the Pbx1-deficient mesDA axons reached into 


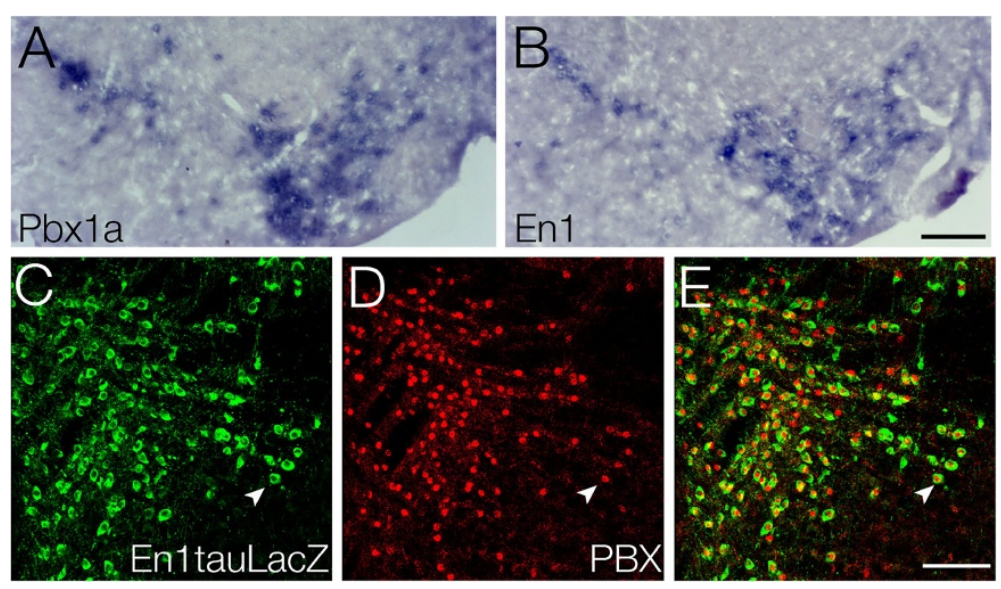

Figure 2 Adult expression of Pbx1a and En1 in mesencephalic dopaminergic neurons. In situ hybridization using digoxigenin-labeled riboprobes against (A) Pbx1a and (B) En1 on adult coronal sections and immunohistochemistry on adult mid-sagittal sections of En $1+/ t \mid Z$ mice using antibodies against (C) pan-Pbx and (D) B-galactosidase to reveal the En1tauLacZ reporter gene. (A, B) Pbx1a and En1 expression co-localize in adult mesDA neurons. (C-E) Pbx1 protein ( $D$, red) is detectable in the nucleus of all En1tauLacZ-expressing ( $C$, green) mesDA neurons ( $E$, arrowheads). Scale bars $A, B=250 \mu \mathrm{m}$ and $C-E=200 \mu \mathrm{m}$. mesDA: mesencephalic dopaminergic.

the ganglionic eminence (data not shown) but the axonal bundle was loosely packed and a small part of the axons had misrouted at the same position in the ventral forebrain where they had stalled at E13 (Figure 4E, F).

Recent studies indicated a role of netrin-1/DCC signaling in the guidance of mesDA axons [35,36], thus, we investigated $P b x 1$ mutant mice for alterations in the expression of netrin-1 and its high affinity receptor $D C C$ [37]. Despite the widespread expression of $P b x 1$ in the telencephalon, netrin-1 expression in the basal ganglia of E14 Pbx1 mutant appeared normal (Figure 5A, B); instead, expression of its receptor $D C C$ [38] was absent in Pbx 1 -deficient mesDA neurons (Figure 5E, F) identified by $\mathrm{TH}$ immunohistochemistry on the parallel sections (Figure 5C, D). To confirm the absence of DCC in mesDA neurons we performed quantitative PCR on E13 ventral midbrain tissue of $P b x 1$ mutant mice compared to littermate controls. We found a reduction of approximately $35-40 \%$ in the expression of $D C C$ in the entire ventral midbrain of $P b x 1$ mutant mice while the expression of netrin-1 was unaltered (Figure 5G).

\section{Analysis of mesencephalic dopaminergic markers in Pbx1 mutants}

In order to assess whether $P b x 1$ deletion leads to the altered expression of other genes associated with mesDA
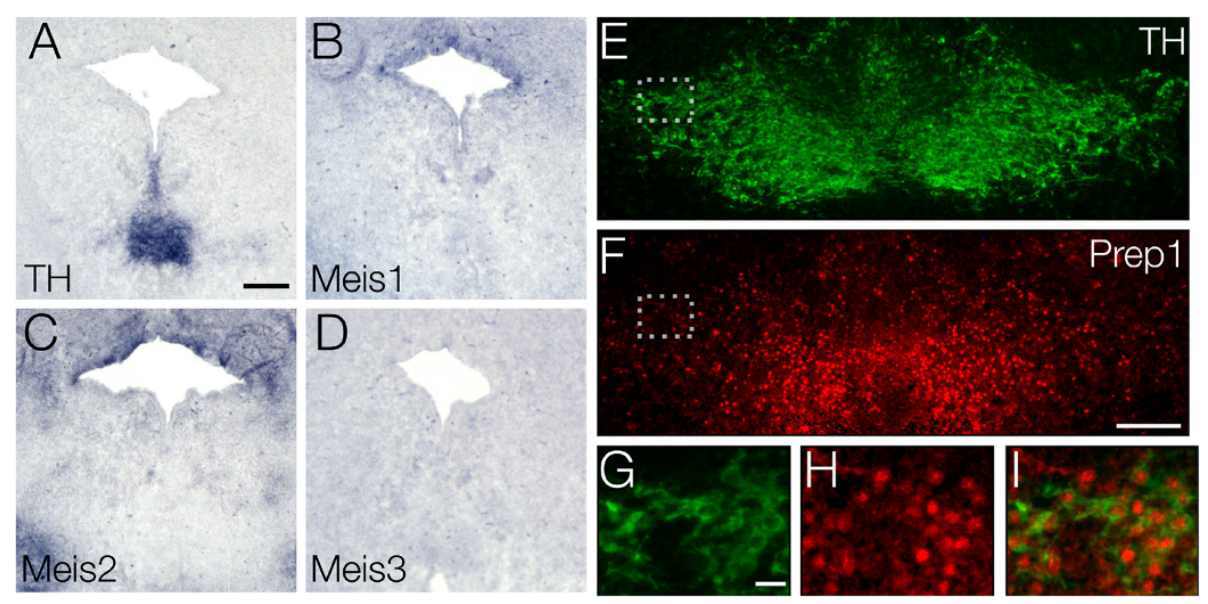

Figure 3 Expression of MEINOX genes in ventral midbrain. In situ hybridization using digoxigenin-labeled riboprobes against (A) $T H$, (B) Meis1, (C) Meis2 and (D) Meis3. Immunohistochemistry using antibodies against TH (E, G) and Prep1 (F, H). (A-D) None of the Meis family members is expressed by E15 mesDA neurons. (E,F) TH (E) and Prep1 (F) are co-expressed in mesDA neurons. (G-I) Magnification of dashed box, showing Prep1-positive nuclei in TH-positive cell body. Dorsal to the top. Scale bars, A-D = $200 \mu \mathrm{m}, \mathrm{E}, \mathrm{F}=250 \mu \mathrm{m}, \mathrm{G}-\mathrm{H}=50 \mu \mathrm{m}$. mesDA: mesencephalic dopaminergic. 

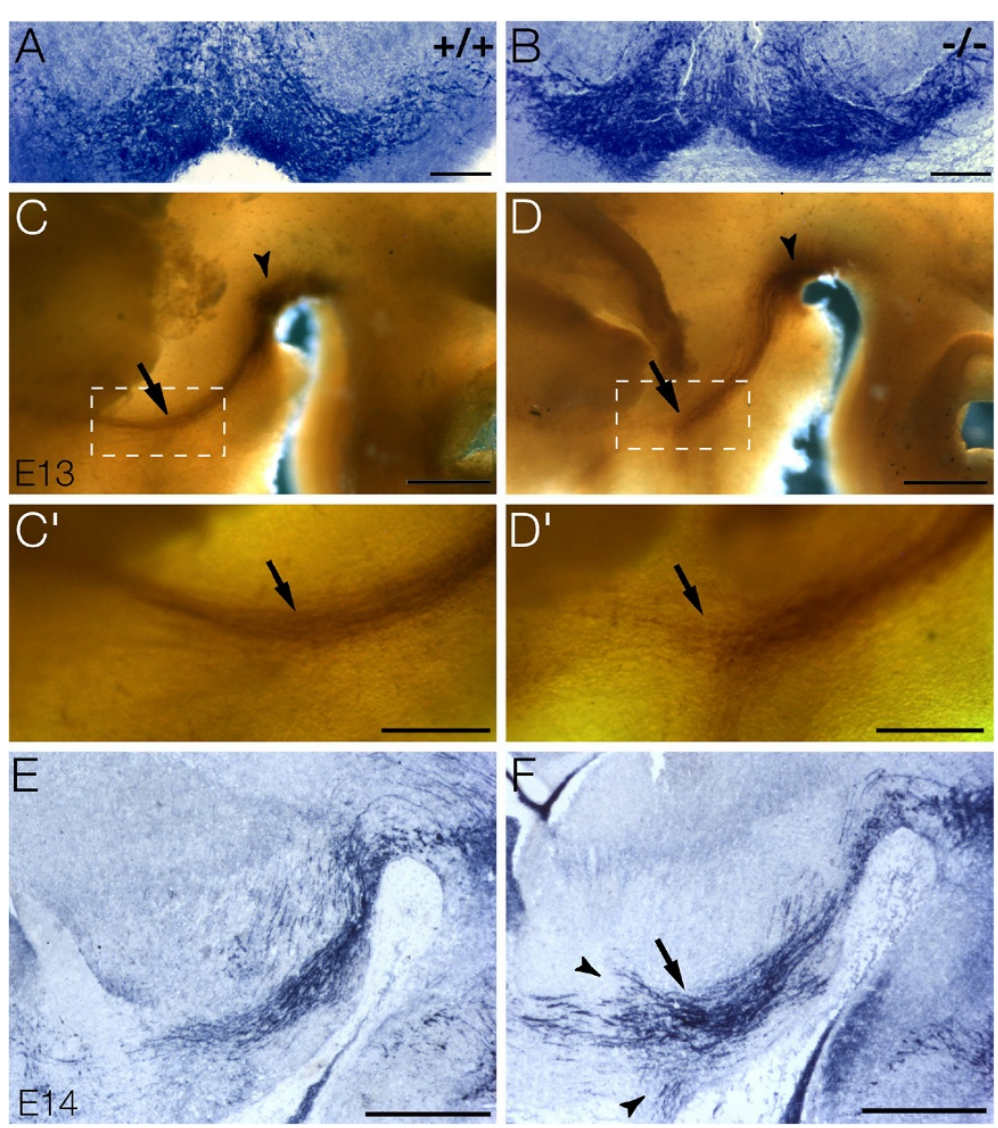

Figure 4 Misrouting of dopaminergic axons in Pbx1-deficient embryos. TH immunohistochemistry on E15 coronal (A, B) and E14 sagittal sections (E, F), and on E13 dissected brains in whole mount preparation (C-D') of wild type (A, C, E) and Pbx1-/- mutant embryos (B, D. F). (A, B) Distribution of mesDA neurons in the ventral midbrain is identical in wild type and Pbx1-/- mice. (C-D') E13 whole mount preparation of isolated neural tube. TH-positive neurons are located in the ventral midbrain (arrowheads) of wild type (C) and Pbx1-deficient (D) embryos. Wild type $\mathrm{TH}$-positive axons have reached deep into the ventral telencephalon, whereas the $\mathrm{Pb} \times 1-/-$ axons have prematurely stopped their growth prior to entry of the ganglionic eminence and begin to defasciculate at the tip (arrow) $\left(D, D^{\prime}\right)$. $C^{\prime}$ and $D^{\prime}$ are higher magnification of the dashed box in C and D. (E, F) At E14, the bundle of TH-positive axons is compact, fasciculated and directed towards the ventral telencephalon in the wild type (E). In the mutants, the axon bundle is wider and disorganized (arrow) and shows misrouted axonal tips (arrowheads) (F). Dorsal to the top, $C-F$ rostral to the left. Scale bars $A, B=200 \mu m, C, D, E, F=500 \mu m$ and $C^{\prime}, D^{\prime}=250 \mu \mathrm{m}$. mesDA: mesencephalic dopaminergic.

neurons phenotype, concomitantly with the perturbation observed in $D C C$ gene expression, we performed in situ hybridization on E15 embryos using probes specific for Nuclear receptor related 1 (Nurr1) [39], En1, En2 [40], LIM homeobox transcription factor 1-beta (Lmx1b) [41], Pituitary homoebox 3 (Pitx3) [42], TH, Dopamine transporter (DAT), Dopa decarboxylase (AADC), Dopamine receptor 2 (DRD2), Ret oncogene (c-ret), Glial cell linederived neurotrophic factor family receptor alpha 1

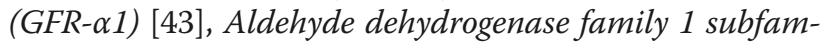
ily A1 (Ahd2) [44] and $\alpha$-synuclein [40]. None of them

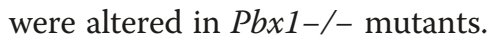

Conversely, the absence of $D C C$ expression in $P b x 1$ deficient mutant embryos suggested that DCC is a direct target of Pbx1. In order to investigate this hypothesis, we searched for putative Pbx1 binding sites in $D C C$ regulatory region by in silico analysis. Our syntenic alignment of human, rat and mouse genomic sequences $20 \mathrm{~kb}$ upstream and $10 \mathrm{~kb}$ downstream of the start codon of the DCC gene in combination with the TRANSFAC database weight matrix for the $P b x 1$ consensus sequence revealed three conserved Pbx1 binding sites in the first intron of $D C C$ at positions A: 775-786, 765-776, 761-772, B: 2059-2067, 2122-2130, 19962004 C: 2584-2592, 2645-2653, 2515-2523 bases downstream of the ATG in human, mouse and rat, respectively (data not shown). However no significant DNA enrichment was achieved by chromatin immunoprecipitation (ChIP) either with a specific Pbx1 antibody or with the pan-Pbx antibody (data not shown), indicating that regulation of $D C C$ expression by $\mathrm{Pbx} 1$ is probably not direct. 

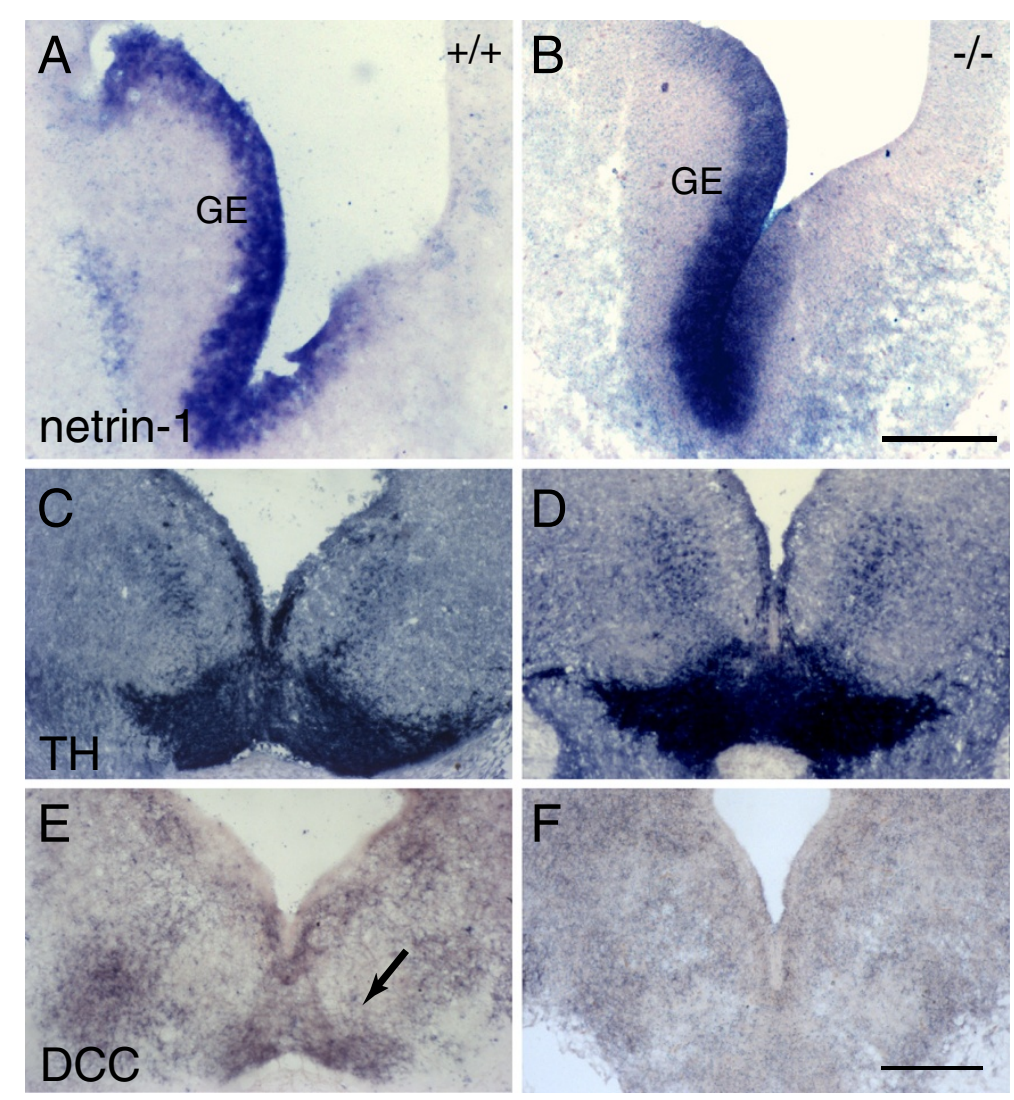

G

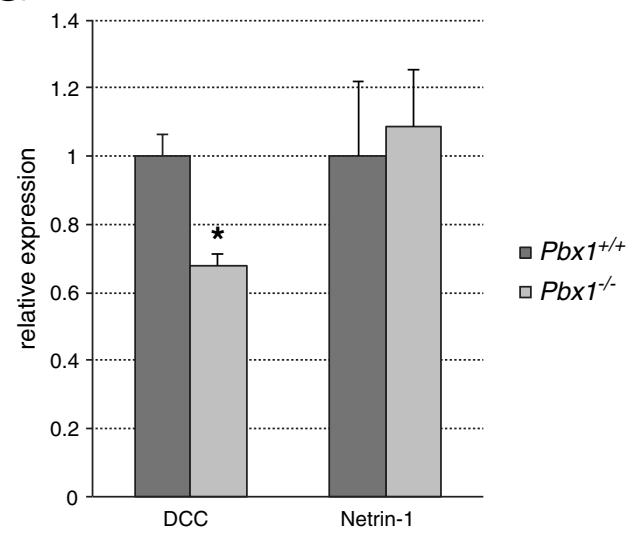

Figure 5 Netrin-1 and DCC expression in Pbx1 null and wild type embryos. In situ hybridization using digoxigenin-labeled riboprobes against netrin-1 ( $A, B)$ and DCC (E, F), immunohistochemistry with antibodies against TH $(C, D)$ on E14 mouse coronal section of wild type (A, $C, E)$ and Pbx1-/- mutants (B, D, F) at level of midbrain. (A, B) Identical expression of netrin-1 in the ganglionic eminence (GE) of wild type (A) and Pbx1-deficient (B) embryos. (C-F) The parallel TH and DCC stained sections reveal DCC expression in wild type mesDA neurons, but no expression in Pbx1-deficient embryos at the same level. Dorsal to the top. Scale bars $=300 \mu \mathrm{m}$. (G) Quantitative PCR using DCC-and netrin-1- specific primers and probes on mRNA extracts from E13.5 ventral midbrain from Pbx $1+/+$ and $P b \times 1-/-$ mutants. Expression levels are relative to the housekeeping gene $R L P L O$, values are expressed as $2^{-\Delta \mathrm{Ct}}$ mean \pm standard error of the mean; $n \geq 4$ for each experiment $\left({ }^{*} P<0.05\right)$. mesDA: mesencephalic dopaminergic.

\section{Discussion}

We show here that Pbxla is expressed in mesDA neurons from E11 into adulthood. During early embryogenesis, its expression in the neural tube is abundant, and becomes later confined in the ventral midbrain to only mesDA neurons. The co-expression of Pbxla and Prep1 in mesDA neurons suggests that Pbx1 nuclear localization is achieved in this neuronal population 
through molecular association with Prep1. We, furthermore, show an aberrant mesDA axonal projection in $P b x 1-/-$ embryos, which is likely the result of the loss of $D C C$ expression. However we were not able to demonstrate direct $\mathrm{Pbx} 1$ binding on the three highly conserved Pbx1 binding sites in the first intron of $D C C$ by ChIP.

A number of studies have shown molecular interactions between Pbx proteins and several other transcription factors and transcriptional co-regulators. The most studied Pbx partners are the Hox proteins. However, $\mathrm{Pbx}$ members form functional heterodimeric complexes with other homeoproteins, such as Engrailed and Pdx1, and other non-homeodomain transcription factors of the basic helix-loop-helix, forkhead and Smad family, as well as with members of the nuclear receptor superfamily $[2,6]$. Pbx loss of function phenotype is very often correlated to the function of the associated partner. $P b x 1$-deficient mice die at E15.5, displaying severe hypoplasia (lungs, liver, stomach, gut, kidneys and pancreas), ectopia (thymus and kidneys) or aplasia (spleen, adrenal gland) of multiple organs, and widespread defects of the axial and appendicular skeleton [7]. Although mice with $P b x 1$ targeted mutation exhibit some degree of homeotic transformations, they do not perfectly resemble mutants for Hox genes, their most studied partners. The same can be said for other Pbx mutants. Pbx3-deficient mice survive to term, but die soon after birth from central respiratory failure [45]. $P b x 1$ and $P b x 3$ have overlapping embryonic expression domains and could therefore exhibit redundant functions. In contrast to $P b \times 1$ - and Pbx3-deficient mice, $P b x 2$-deficient mice are viable and display no apparent phenotype despite its broad expression [46]. Therefore the phenotype of the Pbx targeted mutants could be the result of compensatory functions of other Pbx members and/or partial partner-independent functions $[2,6]$.

The phenotypical alterations in mesDA neurons of Pbx1-deficient mice can be considered in correlation to the well-described Engrailed phenotype in these cells. The targeted deletion of both Engrailed genes leads to severe tissue deletion in the mesencephalon and loss of mesDA neurons at birth [40]. A more detailed analysis of these mutant mice revealed that the dopaminergic neurons are generated in the mesencephalic flexure, but die by E14 without extending axonal processes [15]. MesDA neurons in Pbx1-deficent embryos survive beyond E14 and are able to extend axons; a phenotype that seems to diverge from the complete ablation of mesDA neurons observed in Engrailed double mutant embryos. Yet a cooperative function of Engrailed and Pbx1 cannot be excluded on the base of this sole phenotypic resemblance. Engrailed mutation show a gene-dose dependent effect on the survival of mesDA neurons [40] and no information have been reported about the axonal projections of mesDA neurons in other single or compound Engrailed mutants. Furthermore, our analysis does not exclude a redundant effect of other $P b x$ genes. The presence of $P b x 3$ mRNA expression in these neurons indicates the possibility of a compensatory effect in absence of $P b x 1$, therefore restoring the threshold $\mathrm{Pbx}$ proteins concentration required for a correct development.

We report here that $P b x 1$ loss of function leads to defasciculation and misrouting of mesDA axons in the border between di- and telencephalon. Since $P b x 1$ is expressed in mesDA neurons as well as in the developing target tissue [47], the axonal outgrowth phenotype of Pbx1-deficient mice could reflect alterations in either of the two. The unaltered expression of netrin-1 in the ganglionic eminence, the intact morphology of the tissue and the loss of DCC expression suggest that the mesDA axonal phenotype is likely attributable to a cellautonomous function of Pbx1.

Several studies suggest that multiple cues collaborate to guide dopaminergic axons into a restricted domain through the diencephalon. Initially, migration of mesDA axons rostrally is determined by repulsion from a posterior source of semaphorin. Once in the diencephalon, mesDA axons are constrained in a narrow path established by multiple signals that keep axons from diverging ventrally or dorsally. The ventral boundary requires both Robo/Slit [48,49] and Netrin/DCC [35,36] opposing actions, as both slits repulsion and netrins attraction actions contribute to prevent dopaminergic axons from crossing the midline. Dorsal repulsion instead is likely mediated by attractive cues only, such as netrin and Sonic hedgehog [35,49-51]. Finally, mesDA projections into the basal forebrain and cortex require an unusual attractive activity of semaphorin [52].

A recent analysis of $D C C$ loss of function in vitro and in vivo demonstrated that $D C C$ regulates neuronal precursor cell migration, axon guidance and axonal terminal arborization [36]. Nevertheless, even in absence of $D C C$ expression, mesDA axons are able to reach their target tissue [36]. Differently from the previous report, however, in Pbx1-deficient embryos, loss of DCC expression has no effect on cell migration and seems to affect only long-range axon guidance. In Pbx1-deficent mice, axonal outgrowth is not affected until the mesDA neurons reach the border region between di- and telencephalon, and only at this point does Pbx1-mediated DCC/ netrin signaling seem to be required. Unfortunately, $P b x 1$ mutant mice die at E15.5, preventing further analysis of the phenotype induced by the loss of DCC expression in these mice. No information is available on the embryonic phenotype of DCC mutants to be compared with those of $P b x 1$ mutants. Furthermore, analysis at later stages of the basal forebrain structures affected 
by abnormal nigro-striatal axonal targeting (dorsal striatum, olfactory tubercle, etc...) is not possible in Pbx1-mutants as complete maturation of dopaminergic innervations to the forebrain takes place between E15 and P0 [34,53].

According to the Stein and Tessier-Lavigne 'Hierarchical organization of guidance receptors' model [54], activation of DCC by netrin, and concomitantly of Robo by Slit, leads to silencing of the attractive DCC-mediated netrin response without affecting its growth-stimulatory effect. Indeed, both $D C C$ and Robo are expressed in mesDA neurons at developmental stages consistent with the defect observed in $P b x 1$ mutant embryos and could contribute to the observed phenotype [35,36,49]. Furthermore, a recent study indicated that loss of Slit/Robo signaling leads to widespread errors in mesDA axonal trajectories in the diencephalon, similar to those observed in Pbx1-deficient mice [49].

\section{Conclusions}

In this study, we show that Pbx1 and possibly its cofactor Prep1 are part of the transcriptional factor network that control a key step in mesDA neuronal differentiation by regulating the establishment of mesencephalic-striatal axonal projection. The axon guidance pathways are not just important in development of mesDA neurons they may regulate survival of this neuronal population throughout life, as suggested by genetic linkage studies and their connection to sporadic Parkinson's disease [55,56]. Therefore, Pbx1 may be important in determining the vulnerability of mesDA neurons to degeneration during the early phases of Parkinson's disease.

\section{Methods}

\section{Mutant mice}

Targeted mutation of $P b x 1$ and En1tauLacZ mice has previously been described [7,23]. Pbx1+/- and En1+/tlZ adult mice were crossed into a C57/Bl6 background. The colony was maintained at the central animal facility at the University of Heidelberg. Experiments were carried out in accordance with the European Communities Council Directive of 24 November 1986 (86/609/EEC) for the care and use of experimental animals; all procedures were approved by the central animal facility at the University of Heidelberg. Each of the described phenotypes was found in all analyzed mutant animals $(n \geq 4)$.

\section{In situ hybridization}

Radioactive and digoxigenin in situ hybridizations have been previously described [40]. The riboprobes corresponded to 1644 to 2277 of NM_183355 (Pbx1a), 1917 to 3049 of NM_008783 (Pbx1b), 1468 to 2264 of NM_017463 (Pbx2), 1665 to 2331 of NM_016768 (Pbx3) and 2780 to 3304 of NM_007831 (DCC). TH and En1 are described elsewhere [40].

\section{Immunohistochemistry}

All immunohistochemistry, including the whole mount staining, was performed as described [40] using rabbit and sheep anti-TH antibodies (AB152 and AB1542 EMD Millipore Inc., Billerica, MA, USA) at 1:1,000, rabbit anti-pan-Pbx antibody (sc-888 Santa Cruz Biotechnology Inc., Santa Cruz, California, USA) at 1:2,000, rabbit antiPbx3 antibody (sc-891 Santa Cruz Santa Cruz Biotechnology Inc., Santa Cruz, California, USA) at 1:1,000, goat anti- $ß$-galactosidase at 1:10,000 (Arnel Products Co., New York, NY, USA) and mouse anti-Prep1 antibody at 1:200 (05-766 EMD Millipore Inc., Billerica, MA, USA). The pan-Pbx antibody recognizes a common C-terminal peptide in all of the $50 \mathrm{kDa}$ splice variants of $\mathrm{Pbx} 1, \mathrm{Pbx}-$ 2 and $\mathrm{Pbx} 3$.

\section{Real time PCR}

Quantitative PCRs were performed with a Biorad CFX384 system by using preformulated TaqMan Gene expression assays (Invitrogen, Life Technologies Inc., Carlsbad, California, USA) and calculating the results with the comparative $\mathrm{Ct}$ method. The assays had the following identification tags: Mm00514509_m1 (DCC), Mm00500896_m1 (netrin-1) and Mm01974474_gH (RPLPO). Dissection of ventral midbrain tissue has been previously described [15]. The dissected ventral midbrains were homogenized, the RNA isolated using the RNeasy Mini kit (Qiagen group, USA) and reversetranscribed using the VILO Superscript cDNA synthesis kit (Invitrogen, Life Technologies Inc., Carlsbad, California, USA). Each individual PCR was done in three biological replicates.

\section{In silico promoter analysis}

Syntenic alignment and analysis of transcription factor binding sites of genomic sequences was performed using ECR Browser and rVista2.0 software (http://www.dcode. $\operatorname{org} /$ ). For the identification of transcription factor binding sites, rVista2.0 uses a recently developed method, which combines 'suffix tree'-based fast subsequence search with position weight matrices.

\section{Abbreviations}

ChIP: chromatin immunoprecipitation; DCC: Deleted in colorectal cancer; E: embryonic day; Exd: Extradenticle; Fgf8: Fibroblast growth factor 8; mesDA: mesencephalic dopaminergic; MEINOX: MEIS and KNOX subclass of the TALE superclass; PBC: PBC domain family of the TALE superclass; Pbx: Pre $B$-cell leukemia homeobox; PCR: polymerase chain reaction; TALE: three amino acid loop extension; TH: tyrosine hydroxylase.

\section{Competing interests}

The authors declare that they have no competing interests. 


\section{Acknowledgements}

This work was supported by grants from the German Federal Secretary for Education and Research, BMBF Biofutur 98, the University of Trento and the Michael J. Fox Foundation. We thank Licia Selleri for the Pbx1-/- mice, Martyn Goulding for the En1/tauLacZ mice, Kenneth Campbell and Marc Tessier-Lavigne for the Meis and netrin-1 probes. We also thank Gabi Döderlein and Jiawu Feng for technical help. We thank Francesco Blasi and Luis Fernandez-Diaz for providing us with the Prep1 antibody and Danila Baldessari, Federico Cremisi and Vincenzo Vappavigna for review and critical discussion of the manuscript.

\section{Author details}

'Laboratory of Molecular Neuropathology, Centre for Integrative Biology (CIBIO), University of Trento, Trento, Italy. ${ }^{2}$ Interdisciplinary Center of Neuroscience, Department of Neuroanatomy, University of Heidelberg, Heidelberg, Germany. ${ }^{3}$ Department of Cell and Developmental Biology, Weill Medical College of Cornell University, New York, NY, USA. ${ }^{4}$ Neuroscience Institute, National Research Council (CNR), Pisa, Italy.

\section{Authors' contributions}

PS designed and carried out all the experiments, analyzed and interpreted the data, and drafted the final version of the manuscript. EF and DG provided some experiments, and helped with interpretation of the data and writing of the manuscript. YB helped with interpretation of the data and revised the manuscript. HHS conceived of the study, participated in its design and coordination and drafted the manuscript. All authors read and approved the final manuscript.

Received: 12 May 2012 Accepted: 2 July 2012

Published: 2 July 2012

\section{References}

1. Burglin T: Analysis of TALE superclass homeobox genes (MEIS, PBC, KNOX, Iroquois, TGIF) reveals a novel domain conserved between plants and animals. Nucleic Acids Res 1997, 25:4173-4180.

2. Laurent A, Bihan R, Omilli F, Deschamps S, Pellerin I: PBX proteins: much more than Hox cofactors. Int J Dev Biol 2008, 52:9-20.

3. Lufkin T: Transcriptional control of Hox genes in the vertebrate nervous system. Curr Opin Genet Dev 1996, 6:575-580.

4. Gemel J, Jacobsen C, MacArthur CA: Fibroblast growth factor-8 expression is regulated by intronic engrailed and Pbx1-binding sites. J Biol Chem 1999, 274:6020-6026.

5. Peifer M, Wieschaus E: Mutations in the Drosophila gene extradenticle affect the way specific homeo domain proteins regulate segmental identity. Genes Dev 1990, 4:1209-1223.

6. Moens $C B$, Selleri L: Hox cofactors in vertebrate development. Dev Biol 2006, 291:193-206

7. Selleri L, Depew MJ, Jacobs Y, Chanda SK, Tsang KY, Cheah KS, Rubenstein $J \mathrm{~L}, \mathrm{O}^{\prime}$ Gorman S, Cleary ML: Requirement for Pbx1 in skeletal patterning and programming chondrocyte proliferation and differentiation. Development 2001, 128:3543-3557.

8. Kim SK, Selleri L, Lee JS, Zhang AY, Gu X, Jacobs Y, Cleary ML: Pbx1 inactivation disrupts pancreas development and in Ipf1-deficient mice promotes diabetes mellitus. Nat Genet 2002, 30:430-435.

9. Schnabel C, Selleri L, Cleary M: Pbx1 is essential for adrenal development and urogenital differentiation. Genesis 2003, 37:123-130.

10. Schnabel C, Godin R, Cleary M: Pbx1 regulates nephrogenesis and ureteric branching in the developing kidney. Dev Biol 2003, 254:262-276.

11. Manley NR, Selleri L, Brendolan A, Gordon J, Cleary ML: Abnormalities of caudal pharyngeal pouch development in $\mathrm{Pbx} 1$ knockout mice mimic loss of Hox3 paralogs. Dev Biol 2004, 276:301-312.

12. Brendolan A, Ferretti E, Salsi V, Moses K, Quaggin S, Blasi F, Cleary ML, Selleri $\mathrm{L}: \mathrm{A} \mathrm{Pbx} 1-$ dependent genetic and transcriptional network regulates spleen ontogeny. Development 2005, 132:3113-3126.

13. DiMartino J, Selleri L, Traver D, Firpo M, Rhee J, Warnke R, O'Gorman S, Weissman I, Cleary M: The Hox cofactor and proto-oncogene Pbx 1 is required for maintenance of definitive hematopoiesis in the fetal liver. Blood 2001, 98:618-626.

14. Sgadò P, Albéri L, Gherbassi D, Galasso SL, Ramakers GMJ, Alavian KN, Smidt MP, Dyck RH, Simon HH: Slow progressive degeneration of nigral dopaminergic neurons in postnatal Engrailed mutant mice. Proc Natl Acad Sci USA 2006, 103:15242-15247.

15. Albéri L, Sgadò $P$, Simon HH: Engrailed genes are cell-autonomously required to prevent apoptosis in mesencephalic dopaminergic neurons. Development 2004, 131:3229-3236.

16. Davis CA, Joyner AL: Expression patterns of the homeo box-containing genes En-1 and En-2 and the proto-oncogene int-1 diverge during mouse development. Genes Dev 1988, 2:1736-1744.

17. Joyner AL, Herrup K, Auerbach BA, Davis CA, Rossant J: Subtle cerebellar phenotype in mice homozygous for a targeted deletion of the En-2 homeobox. Science 1991, 251:1239-1243.

18. Gosgnach S, Lanuza GM, Butt SJB, Saueressig H, Zhang Y, Velasquez T, Riethmacher D, Callaway EM, Kiehn O, Goulding M: V1 spinal neurons regulate the speed of vertebrate locomotor outputs. Nature 2006, 440:215-219.

19. Liu A, Joyner AL: EN and GBX2 play essential roles downstream of FGF8 in patterning the mouse mid/hindbrain region. Development 2001, 128:181-191.

20. Alavian $\mathrm{KN}$, Scholz C, Simon HH: Transcriptional regulation of mesencephalic dopaminergic neurons: the full circle of life and death. Mov Disord 2008, 23:319-328.

21. Erickson T, Scholpp S, Brand M, Moens CB, Waskiewicz AJ: Pbx proteins cooperate with Engrailed to pattern the midbrain-hindbrain and diencephalic-mesencephalic boundaries. Dev Biol 2007, 301:504-517.

22. Wagner K, Mincheva A, Korn B, Lichter P, Popperl H: Pbx4, a new Pbx family member on mouse chromosome 8 , is expressed during spermatogenesis. Mech Dev 2001, 103:127-131.

23. Saueressig H, Burrill J, Goulding M: Engrailed-1 and netrin-1 regulate axon pathfinding by association interneurons that project to motor neurons. Development 1999, 126:4201-4212.

24. Capdevila J, Tsukui T, Rodriquez Esteban C, Zappavigna V, Izpisua Belmonte JC: Control of vertebrate limb outgrowth by the proximal factor Meis2 and distal antagonism of BMPs by Gremlin. Mol Cell 1999, 4:839-849.

25. Gonzalez-Crespo S, Morata G: Genetic evidence for the subdivision of the arthropod limb into coxopodite and telopodite. Development 1996, 122:3921-3928.

26. Morata G: How Drosophila appendages develop. Nat Rev Mol Cell Biol 2001, 2:89-97.

27. Ryoo HD, Marty T, Casares F, Affolter M, Mann RS: Regulation of Hox target genes by a DNA bound Homothorax/Hox/Extradenticle complex. Development 1999, 126:5137-5148.

28. Abu-Shaar M, Ryoo H, Mann R: Control of the nuclear localization of Extradenticle by competing nuclear import and export signals. Genes Dev 1999, 13:935-945

29. Berthelsen J, Kilstrup-Nielsen C, Blasi F, Mavilio F, Zappavigna V: The subcellular localization of PBX1 and EXD proteins depends on nuclear import and export signals and is modulated by association with PREP1 and HTH. Genes Dev 1999, 13:946-953.

30. Mercader N, Leonardo E, Azpiazu N, Serrano A, Morata G, Martinez C, Torres $\mathrm{M}$ : Conserved regulation of proximodistal limb axis development by Meis1/Hth. Nature 1999, 402:425-429.

31. Jaw TJ, You LR, Knoepfler PS, Yao LC, Pai CY, Tang CY, Chang LP, Berthelsen J, Blasi F, Kamps MP, Sun YH: Direct interaction of two homeoproteins, homothorax and extradenticle, is essential for EXD nuclear localization and function. Mech Dev 2000, 91:279-291.

32. Kilstrup-Nielsen C, Alessio M, Zappavigna V: PBX1 nuclear export is regulated independently of PBX-MEINOX interaction by PKA phosphorylation of the PBC-B domain. EMBO J 2003, 22:89-99.

33. Toresson $\mathrm{H}$, Parmar M, Campbell K: Expression of Meis and Pbx genes and their protein products in the developing telencephalon: implications for regional differentiation. Mech Dev 2000, 94:183-187.

34. Hu Z, Cooper M, Crockett D, Zhou R: Differentiation of the midbrain dopaminergic pathways during mouse development. J Comp Neurol 2004, 476:301-311.

35. Lin L, Rao Y, Isacson O: Netrin-1 and slit-2 regulate and direct neurite growth of ventral midbrain dopaminergic neurons. Mol Cell Neurosci 2005, 28:547-555.

36. Xu B, Goldman JS, Rymar W, Forget C, Lo PS, Bull SJ, Vereker E, Barker PA, Trudeau LE, Sadikot AF, Kennedy TE: Critical roles for the netrin receptor deleted in colorectal cancer in dopaminergic neuronal precursor 
migration, axon guidance, and axon arborization. Neuroscience 2010, 169:932-949.

37. Kennedy TE: Cellular mechanisms of netrin function: long-range and short-range actions. Biochem Cell Biol 2000, 78:569-575.

38. Livesey FJ, Hunt SP: Netrin and netrin receptor expression in the embryonic mammalian nervous system suggests roles in retinal, striatal, nigral, and cerebellar development. Mol Cell Neurosci 1997, 8:417-429.

39. Zetterstrom RH, Williams R, Perlmann T, Olson L: Cellular expression of the immediate early transcription factors Nurr1 and NGFI-B suggests a gene regulatory role in several brain regions including the nigrostriatal dopamine system. Brain Res Mol Brain Res 1996, 41:111-120.

40. Simon HH, Saueressig H, Wurst W, Goulding MD, O'Leary DD: Fate of midbrain dopaminergic neurons controlled by the engrailed genes. J Neurosci 2001, 21:3126-3134.

41. Smidt M, Asbreuk C, Cox J, Chen H, Johnson R, Burbach J: A second independent pathway for development of mesencephalic dopaminergic neurons requires Lmx1b. Nat Neurosci 2000, 3:337-341.

42. Smidt M, Van Schaick H, Lanctot C, Tremblay J, Cox J, Van DKA, Wolterink G, Drouin J, Burbach J: A homeodomain gene Ptx3 has highly restricted brain expression in mesencephalic dopaminergic neurons. Proc Natl Acad Sci U S A 1997, 94:13305-13310.

43. Nosrat C, Tomac A, Hoffer B, Olson L: Cellular and developmental patterns of expression of Ret and glial cell line-derived neurotrophic factor receptor alpha mRNAs. Exp Brain Res 1997, 115:410-422.

44. McCaffery P, Drager UC: High levels of a retinoic acid-generating dehydrogenase in the meso-telencephalic dopamine system. Proc Natl Acad Sci U S A 1994, 91:7772-7776.

45. Rhee JW, Arata A, Selleri L, Jacobs Y, Arata S, Onimaru H, Cleary ML: Pbx3 deficiency results in central hypoventilation. Am J Pathol 2004, 165:1343-1350.

46. Selleri L, DiMartino J, van Deursen J, Brendolan A, Sanyal M, Boon E, Capellini T, Smith KS, Rhee J, Pöpperl H, Grosveld G, Cleary ML: The TALE homeodomain protein $\mathrm{Pbx} 2$ is not essential for development and long-term survival. Mol Cell Biol 2004, 24:5324-5331.

47. Roberts $\mathrm{V}$, van Dijk M, Murre C: Localization of Pbx1 transcripts in developing rat embryos. Mech Dev 1995, 51:193-198.

48. Bagri A, Marín O, Plump AS, Mak J, Pleasure SJ, Rubenstein JLR, TessierLavigne M: Slit proteins prevent midline crossing and determine the dorsoventral position of major axonal pathways in the mammalian forebrain. Neuron 2002, 33:233-248.

49. Dugan JP, Stratton A, Riley HP, Farmer WT, Mastick GS: Midbrain dopaminergic axons are guided longitudinally through the diencephalon by Slit/Robo signals. Mol Cell Neurosci 2011, 46:347-356.

50. Comoletti D, De Jaco A, Jennings LL, Flynn RE, Gaietta G, Tsigelny I, Ellisman MH, Taylor P: The Arg451Cys-neuroligin-3 mutation associated with autism reveals a defect in protein processing. J Neurosci 2004, 24:4889-4893

51. Hammond R, Blaess S, Abeliovich A: Sonic hedgehog is a chemoattractant for midbrain dopaminergic axons. PLoS One 2009, 4:e7007.

52. Kolk SM, Gunput R-AF, Tran TS, van den Heuvel DMA, Prasad AA, Hellemons AJCGM, Adolfs Y, Ginty DD, Kolodkin AL, Burbach JPH, Smidt MP, Pasterkamp RJ: Semaphorin $3 \mathrm{~F}$ is a bifunctional guidance cue for dopaminergic axons and controls their fasciculation, channeling, rostral growth, and intracortical targeting. J Neurosci 2009, 29:12542-12557.

53. Vives J, Sasajala P, Chang KH, Zhao S, Li M: A mouse model for tracking nigrostriatal dopamine neuron axon growth. Genesis 2008, 46:125-131.

54. Stein $\mathrm{E}$, Tessier-Lavigne M: Hierarchical organization of guidance receptors: silencing of netrin attraction by slit through a Robo/DCC receptor complex. Science 2001, 291:1928-1938.

55. Lesnick TG, Papapetropoulos S, Mash DC, Ffrench-Mullen J, Shehadeh L, de Andrade M, Henley JR, Rocca WA, Ahlskog JE, Maraganore DM: A genomic pathway approach to a complex disease: axon guidance and Parkinson disease. PLoS Genet 2007, 3:e98.

56. Lin L, Lesnick TG, Maraganore DM, Isacson O: Axon guidance and synaptic maintenance: preclinical markers for neurodegenerative disease and therapeutics. Trends Neurosci 2009, 32:142-149.

doi:10.1186/1749-8104-7-24

Cite this article as: Sgadò et al:: The atypical homeoprotein Pbx1a participates in the axonal pathfinding of mesencephalic dopaminergic neurons. Neural Development 2012 7:24.

\section{Submit your next manuscript to BioMed Central and take full advantage of:}

- Convenient online submission

- Thorough peer review

- No space constraints or color figure charges

- Immediate publication on acceptance

- Inclusion in PubMed, CAS, Scopus and Google Scholar

- Research which is freely available for redistribution 